\title{
REALIZACIÓN DE CRITERIOS DE VALORACIÓN SOBRE EL TRABAJO POR PROYECTOS A PARTIR DE LA OBSERVACIÓN DIRECTA DE LA METODOLOGIA EN UN CASO REAL DE UN AULA DE EDUCACIÓN INFANTIL
}

\author{
Ángela Gago Morate \\ Universidad de Valladolid \\ agagomorate@gmail.com \\ Deilis Ivonne Pacheco Sanz \\ Universidad de Valladolid \\ deilisivonne.pacheco@uva.es \\ Alejandro Canedo García \\ Universidad de León \\ acang@unileon.es \\ Beatriz Martín Sánchez \\ Universidad de Valladolid \\ beamarsan7@hotmail.com \\ Yvet Bleye Varona \\ Universidad de Valladolid \\ yvet.b@hotmail.com
}

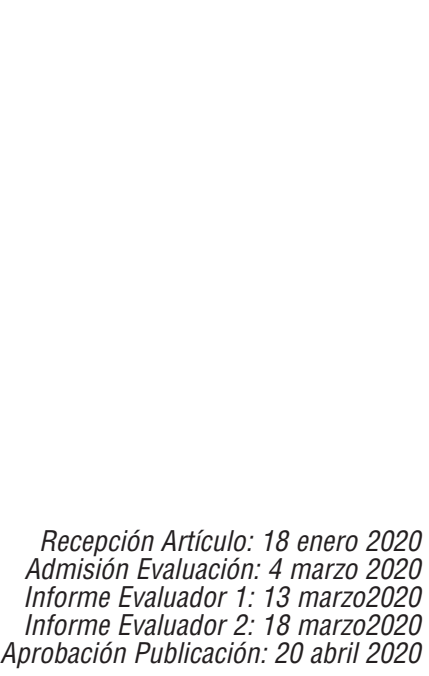

\section{RESUMEN}

Los estudios de formación para la docencia, en Educación Primaria y Educación Infantil, han permitido conocer diferentes metodologías y modelos educativos, así como el aprendizaje de destrezas, actitudes y competencias profesionales que se exigen para ejercer la docencia. En el caso de la metodología del Trabajo por Proyectos, a pesar de tener su origen hace varios siglos, es actualmente cuando está en auge en España, implementándose sobre todo en las aulas de Educación Infantil, por lo que se ha decidido ampliar la información sobre esta metodología. Por ello, ha sido necesario recurrir a la revisión de diversos estudios y ejemplos de aplicaciones prácticas, ejecutadas en algunos centros educativos. El criterio de selección del colegio para este estudio, estuvo enmarcado dentro del contexto de la elección de centros para el desarrollo del Practicum III en la Universidad de Valladolid, tras la investigación previa de aquellos centros donde trabajaban y llevaban a cabo esta metodología. Es así cómo la escogencia del colegio, permitió tanto la observación directa sobre la práctica docente en un aula de Educación Infantil con 22 estudiantes de 4 años, así como realizar una evaluación de la aplicación práctica de la Metodología del Trabajo por Proyectos, bajo el foco del siguiente planteamiento: ¿se están cumpliendo los criterios que sigue esta metodología? Durante la práctica observada, se fueron obteniendo datos y se construyeron 
8 criterios que permiten valorar la práctica observada. Asimismo, se ha realizado una propuesta de mejora sustentada en lo que la autora de este trabajo ha contrastado con los principios metodológicos para la aplicación del Trabajo por Proyectos, siguiendo un orden lógico de acuerdo a los criterios de evaluación propuestos y respondiendo a: ¿Qué dice la teoría?, ¿Qué se observó en la práctica real del aula? y 'la propuesta de mejora: ¿Qué haría yo?

Palabras clave: metodología; trabajo por proyectos; educación infantil; observación; criterios de evaluación; propuesta de mejora

\begin{abstract}
Development of assessment criteria for project work, based on direct observation of the methodology, in a real case of an infant education classroom. The studies of training for teaching, in Primary Education and Early Childhood Education, have allowed to know different methodologies and educational models, as well as the learning of professional skills, attitudes and competences that are required to practice teaching. In the case of Project Work methodology, despite having its origin several centuries ago, it is currently booming in Spain, being implemented especially in Early Childhood education classrooms, so it has been decided to expand the information on this methodology. For this reason, it has been necessary to resort to the review of various studies and examples of practical applications, carried out in some educational centers. The selection criteria of the school selected for this study, was framed within the context of the choice of centers for the development of Practicum III at the University of Valladolid, after prior investigation of those centers where this methodology was worked and carried out. This is how the choice of school allowed both direct observation of teaching practice in an Early Childhood classroom with 22 4-year-old students, as well as an evaluation of the practical application of the Project Work Methodology, under the focus of the following approach: are the criteria that this methodology follows being met? During the observed practice, data were obtained and 8 criteria were constructed to assess the observed practice. Likewise, a proposal for improvement has been made based on what the author of this work has contrasted with the methodological principles for the application of Project Work, following a logical order according to the proposed evaluation criteria and responding to: What says the theory?, What was observed in the real practice of the classroom? and 'the improvement proposal: What would I do?

Keywords: methodology; project work; early childhood education; observation; evaluation criteria; proposal for improvement
\end{abstract}

\title{
ANTECEDENTES
}

La educación ha tenido una gran evolución a lo largo del tiempo, Ilevando a cabo una serie de cambios considerados beneficiosos para el alumnado. Dentro de estos cambios, se encuentran aquellos que tienen que ver con los nuevos enfoques de las metodologías que se están llevando a cabo en los Centros educativos y, por ende, en los diferentes niveles educativos.

Haciendo un balance sobre las diferentes metodologías activas que les están dando mayores usos por su aplicabilidad, se encuentra que el Trabajo por Proyectosresponde a las nuevas expectativas de la sociedad actual, pues diversos autores lo considerancomo un método pedagógico en el que el protagonista principal es el alumnado. Por ello, la metodología exige un esfuerzo por parte del profesorado, en cuanto a las fases que deben seguir para su ejecución, y por parte del resto de los agentes implicados, como el alumnado y las familias, en cuanto a sentirse sujetos y no objetos del proceso educativo (Arias y Rial, 2009; Domínguez, 2000; Díez Navarro, 1998; Martín García, 2006).

\section{OBJETIVOS DE LA INVESTIGACIÓN}

El objetivo finalse centró en elaborar un análisis sobre la aplicación del Trabajo por Proyectos en un caso real de aula, a partir de la propuesta de criterios que permitan valorar su ejecución práctica. Para ello, se hizo una revisión y análisis teórico de los diferentes principios metodológicos que fundamentan esta metodología, con- 
trastada con la observación directa y sistemática del aula. Para poder conseguir dicho objetivo, se hizo necesario cumplir con algunos objetivos específicos, por ejemplo: i) reflexionar sobre el rol de los diferentes agentes implicados en la metodología, ii) describir la puesta en práctica de un trabajo por proyectos en un contexto real de aula. Dichos objetivos, facilitaron tanto la construcción de criterios para el análisis de la aplicación práctica, así como diferentes propuestas de mejora como futura docente.

\section{METODOLOGÍA}

La metodología en la que se ha basado este trabajo sigue la secuencia indagación/observación/propuesta. Dentro de ésta, se ha realizado una toma de datos sobre los puntos que llamaron la atención durante la observación de la aplicación práctica de la metodología, durante la experiencia educativa de las practicas docentes desarrollada en un colegio de la Comunidad Autónoma de Castilla y León, en el cual se trabajaba mediante dicha metodología. Este modo de proceder, condujo a realizar una evaluación sobre la práctica docente, en cuanto a la manera de llevar a cabo este tipo de metodología. Estos rasgos o puntos sobresalientes son los que a partir de ahora se llaman 'criterios'.

¿Cómo surgen o cuál fue el proceso de construcción de los criterios o categorías de análisis para la valoración del trabajo por proyectos?

A partir del momento en el que se establece contacto con la situación educativa de un aula de infantil, con niños de 4 años, aumentó el interés por indagar sobre las diferentes definiciones, las fases que deben cumplir los Proyectos, el papel de los agentes que participan en esta metodología y otros aspectos considerados importantes, para tener una base sólida en la cual fundamentar la valoración o evaluación de la práctica docente, objeto de estudio.

El proceso que permitió determinar los criterios, que son claves para la evaluación del Trabajo por Proyectos, fue el siguiente: después de la fase de recogida de datos dentro del aula, a través de la observación sistemáticade la práctica docentedurante el tiempo de ejecución del Practicum, se contrastaron esos datos con la teoría investigada sobre este método (principios metodológicos); luego, se pasó a organizar toda la información, dividiéndola en los diferentes puntos que caracterizan al Trabajo por Proyectos y destacando la idea central de cada uno con un nombre o título. De esta manera, se obtuvo la propuesta de cinco criterios, tal y como se muestran en la tabla 1. 
Tabla 1. Propuesta de criteriospara la evaluación del Trabajo por Proyectos

\begin{tabular}{cl}
\hline CRITERIOS / Autor(es) & \multicolumn{1}{c}{ IDEA CENTRAL } \\
\hline Intereses y/o motivación del alumnado & -El tema debe ser elegido entre un acuerdo \\
(Arias, y Rial, 2009) & $\begin{array}{l}\text { docente-alumnado. } \\
\text {-El docente tiene el rol de que el alumnado } \\
\text { proponga ideas con el fin de elegir el tema } \\
\text { entre ambos. }\end{array}$ \\
Implicación de las familias & -El papel de las familias tiene mucho peso. \\
(Domínguez, 2000) & -La escuela necesita colaboración por \\
& parte de las familias, aportando \\
información y materiales al proyecto. \\
Fases del trabajo por proyectos & -Son tres grupos los que componen las \\
(Díez Navarro, 1998) & fases del Trabajo por Proyectos: i) qué \\
& sabemos sobre el tema, ii) qué queremos \\
Trabajo interdisciplinario y/o & saber, iii) qué hemos aprendido. \\
cooperativo entre docentes & -La coordinación entre los docentes de \\
(Martín García, 2006) & Educación Infantil es importante a la hora \\
de organizar y realizar los proyectos.
\end{tabular}

Fuente: elaboración propia.

\section{MUESTRA Y/O PARTICIPANTES}

Los participantes de este estudio, hacen referencia a un grupo heterogéneo de 22 niños y niñas. Entre ellas, destacamos a dos niñas con necesidades educativas especiales. Una de ellas, tiene una 'enfermedad rara' cuya principal característica es el bajo tono muscular acompañado de problemas cognitivos. El otro caso consiste en dificultades tanto motrices como cognitivas, causado por un accidente siendo bebé. Dentro del aula, también existía un grupo de 4-5 varones que, pese a ser cognitivamente muy competentes tenían problemas para acatar normas, lo que perturbaba el clima de la clase. Para finalizar, destacar un grupo mixto que respondían muy bien tanto a nivel conductual como cognitivo y que, además, cuentan con el refuerzo por parte de sus familias; y, otros dos casos de niñas con procedencia marroquí.

\section{RESULTADOS}

Los resultados obtenidos, giran en torno a la contrastación de la práctica observada con los criterios propuestos para la evaluación de dicha práctica. El esquema seguido fue el siguiente: i) ¿qué dice la teoría? (ver tabla 1); ii) ¿qué se observó en la práctica real? (ver tabla 2). Posterior a esta valoración, se hace una propuesta de mejora en la que se explica de qué manera se podrían Ilevar a cabo estos criterios, en la práctica profesional futura (ver tabla 3).

En cuanto a la metodología docente observada, siguiendo el Proyecto Educativo de Centro (PEC) en Educación Infantil, la misma debíaestar basada en un enfoque constructivista. Con dicho enfoque, se debe impulsar el aprendizaje a través de la motivación, atención a la diversidad y sensibilización en valores. El trabajo del ciclo de Infantil, se fundamenta en un trabajo por proyectos comunes, constituyendo el eje principal sobre el que se sustenta todo el trabajo dentro del aula. No obstante, en el aula objeto de observación, la tutora no demostró 
ser coherente con la teoría del enfoque constructivista, pues en todo momento daba respuestas directas sobre problemas o conflictos, sin ofertar las herramientas y oportunidades para que el alumnado supiera cómo solucionarlos. De esta manera, el alumnado dista mucho de construir el aprendizaje con sus compañeros (como nota de observación de un trabajo con un enfoque meramente tradicional, se destaca que la tutora utilizaba sellos de diferentes colores -verde, amarillo y rojo-, como estrategias de calificación de los trabajos del alumnado). En la tabla 2, se describen los resultados más relevantes, obtenidosde la práctica docente observada, de acuerdo a los criterios propuestos para evaluar el Trabajo por Proyectos.

Tabla 2. Descripción de la práctica real observada, de acuerdo a los criterios propuestos para evaluar el Trabajo por Proyectos

CRITERIOS ¿Qué se observó en la práctica real?

Intereses y/o motivación del alumnado -Los proyectos que realizan no parten de los intereses del alumnado, sino que sonelegidos y determinados por el ciclo de Ed. Infantil a principio de curso. Por lo tanto, el proyecto no está basado en el interés del alumnado.

Implicación de las familias

Fases del trabajo por proyectos

Trabajo interdisciplinario y/o
cooperativo entre docentes

Trabajo interdisciplinario y/o cooperativo entre las áreas de educación infantil
-La implicación de las familias fue alta; sin embargo, las actividades que llevaban a cabo en el aula no estaban acordes con el nivel del alumnado.

-No se han llevado a cabo las fases que exige la metodología del trabajo por proyectos

-Existe poca coordinación entre las docentes del ciclo de Educación Infantil en relación a los materiales trabajados.

-En la práctica observada no se dio un enfoque globalizador.

-Los proyectos se trabajaban como Unidades Didácticas.

-No se ha trabajado el proyecto en las diferentes áreas de la etapa.

Fuente: Elaboración propia

Como bien se detecta en la tabla 2, existe un marcado contraste entre la teoría investigada con la realidad observada dentro del aula.

Tras la valoración descriptiva de los resultados obtenidos, se hace una propuesta de mejora en la que se explica de qué manera se podrían llevar a cabo estos criterios, en la práctica profesional futura. Esta propuesta de mejora, ha surgido luego de haber seguido un proceso de contraste entre la teoría investigada con la realidad observada dentro del aula. De esta manera, se puede ver un pequeño resumen de cada propuesta en la Tabla 3. 
Tabla 3 Propuesta de mejora en relación a los criterios

\begin{tabular}{|c|c|}
\hline CRITERIOS / Autor(es) & PROPUESTA DE MEJORA \\
\hline Intereses y/o motivación del alumnado & $\begin{array}{l}\text {-Ofrecer la oportunidad que el proyecto } \\
\text { parta de sus propios intereses. } \\
\text {-Esto se puede hacer de dos formas: modo } \\
\text { explícito (se realiza una lluvia de ideas y } \\
\text { el alumnado propone temas); ó modo } \\
\text { implícito (el docente propone una serie de } \\
\text { temas y el alumnado tiene la opción de } \\
\text { elegir cuál quiere trabajar). } \\
\text {-El docente será consciente de que en esta } \\
\text { metodología el principal protagonista es } \\
\text { el alumnado. } \\
\text {-Siendo eldocente la mejor persona que } \\
\text { conoce el aula, es importante que realice } \\
\text { una reunión o papel informativo, antes de } \\
\text { empezar cada } \\
\text { proponiendoactividades que considera } \\
\text { adecuadas al nivel del aula. } \\
\text {-Estas actividades/experimentos estarán } \\
\text { realizados en base a los intereses y } \\
\text { necesidades del alumnadopara que, de } \\
\text { esta forma, las familias conozcan de dónde } \\
\text { parte el alumnado y en qué línea sería } \\
\text { positivo continuar. }\end{array}$ \\
\hline Fases del trabajo por proyectos & $\begin{array}{l}\text {-Organizar las rutinas incluyendo en ellas } \\
\text { las diferentes fases del Proyecto de la } \\
\text { siguiente manera: }\end{array}$ \\
\hline
\end{tabular}

- Qué sabemos: Se realizará una lluvia de ideas que se pueden llevar a cabo en la asamblea, después del recreo, buscando un momento en el que haya un clima de confianza para fomentar la expresión oral. Las ideas aportadas, se pueden escribir en una cartulina.

- Qué queremos saber: Se llevará a cabo una lluvia de ideas en la que el alumnado deberá aportar aquello que le interesa saber sobre el tema. En una cartulina diferente, se escribirán las ideas que se han recogido. Al finalizar esta fase, es importante dar la información necesaria a las familias en un papel para que investiguen sobre ello. Cada alumno tendrá una fecha en la que tiene que presentar la informacióna sus compañeros. 


\section{Trabajo interdisciplinario y/o cooperativo entre docentes}

\section{Trabajo interdisciplinario y/o} cooperativo entre las áreas de educación infantil
- Qué hemos aprendido: Al final del proyecto, se haráuna conclusión del mismo. Un agrupamiento que favorecería este intercambio de ideas podría ser el mismo que se lleva a cabo en la asamblea. Esta fase nos sirve para contrastar el panel de la primera fase, con lo que se ha aprendido durante el proyecto.

-Al final del proyecto, se realizará un mural con toda la información recogida durante el mismo.

-Siendo la coordinación entre docentes tan importante en el proceso, lo que se propone es que sean ellos quienes determinen los grados de dificultad, así como la organización y precisión de los objetivos de cada nivel.

-Además, se propone realizar actividades en común sobre el Proyecto, tal y como realizaban los talleres internivelares (juntando las tres clases de Infantil; 3,4 y 5 años para realizar actividades comunes).

-Después de haber determinado las 3 fases que exige la metodología, se elaboraría el material necesario para cumplir con lo que el alumnado quiere aprender. Este material será elaborado en torno a las inquietudes y los intereses del alumnado. -Realizar una reunión con los docentes que imparten el resto de áreas, para conseguir un enfoque globalizador.

Fuente: Elaboración propia

\section{CONCLUSIONES/DISCUSIÓN}

Gracias a la realización de una revisión bibliográfica se ha conseguido tener una visión más global sobre diferentes metodologías y, en especial, sobre el Trabajo por Proyectos.

Tal y como podemos ver en los resultados obtenidos observamos que la teoría investigada sobre el Trabajo por Proyectos dista mucho de lo que ha sido la realidad observada dentro del aula descrito.

La observación, la toma de datos, la reflexión, los ciclos de investigación-observación y el hecho de darle vueltas del mismo modo, pero con criterios diferentes ha resultado muy enriquecedor durante el proceso. Esta manera de organizarlo nos ha ayudado para personalizar este trabajo, pero también para poder realizar un análisis cercano de la práctica, así como para elaborar las propuestas de mejora.

Realizar las propuestas de mejora nos ha resultado positivo ya que ha exigido un gran proceso de reflexión y comparación constante entre la teoría investigada y la práctica docente, con el fin de trasladar dicha propuesta a una futura clase de Educación Infantil. Al haber realizado la propuesta de dicho modo, hemos podido acercarnos 
a aspectos más concretos y basados en una realidad como puede ser tener en cuenta a los agentes implicados en este proceso (docente, alumnado, familia), siendo conscientes de la necesaria vinculación entre los mismos.

Para concluir, destacamos que, en esta metodología, aunque el principal protagonista sea el alumnado (con la necesaria colaboración de la familia, en estas edades), el docente tiene que ofrecer los pasos a los alumnos que les ayude a preguntarse aspectos de la realidad, a investigar, a indagar, a observar, a trabajar en equipo, a exponer y a aprender de sus compañeros y de sus propios errores. Por este motivo el trabajo del docente tiene un gran peso que también se verá reflejado en la vida futura del alumnado.

\section{REFERENCIAS BIBLIOGRÁFICAS}

Arias, A.; Arias, D.; Navaza, V. y Rial, D. (2009). O traballo por proxectos. Santiago: Xunta de Galicia.

Díez navarro. (1998). C: La oreja verde de la escuela. Trabajo por proyectos y vida cotidiana en la escuela infantil. Ediciones de la Torre.

Domínguez Chillón, G. (2000). Proyectos de trabajo. Una escuela diferente. Colección Aula Abierta. Madrid: La Muralla, S.A.

Escamilla, A. (2009). Las competencias en la programación de aula. Infantil y primaria (3-12 años). Barcelona: Graó.

García-Ruíz, R. (2013). Enseñar y aprender en Educación Infantil a través de proyectos. Santander: Editorial de la Universidad de Cantabria.

Martín García, X. (2006). "Investigar y aprender. Cómo organizar un proyecto”. Barcelona. ICE Universitat de Barcelona. Horsori Editorial.

Parra, J. M. (2005). La Educación Infantil: su dimensión didáctica y organizativa. . Granada: Grupo Editorial Universitario. 